2 weeks. Extrapolating from this, there could be over 30,000 goldfinches, 7,000 serins and 4,500 linnets sold per year in the 21 weekly markets we visited. The birds sold in the hundreds of pet shops around the country, the weekly markets we did not visit, and those sold online would add substantially to this number.

Vendors reported that goldfinches are becoming increasingly difficult to source from the wild but that there is no lessening of demand. Already, the pressure has shifted from the Algerian to Moroccan populations because of the difficulty in securing sufficient numbers of birds. The illegal songbird trade in Algeria is threatening wild populations of European goldfinches across North Africa and potentially threatening other species of finch. We therefore recommend that the Algerian government enforce its wildlife trade laws to ensure the conservation of these, and other, species.

Daniel Bergin and Vincent NiJMan Oxford Wildlife Trade Research Group, Oxford, UK. E-mail vnijman@brookes.ac.uk

SADEK ATOUSSI Université 8 mai 1945 Guelma, Guelma, Algeria

\section{Evidence to action: research to address illegal wildlife trade}

The Oxford Martin Programme on the Illegal Wildlife Trade (illegalwildlifetrade.net) has launched a key research brief, Evidence to Action: Research to Address Illegal Wildlife Trade (osf.io/preprints/socarxiv/35ndz). This brief, addressed to policy makers and practitioners, outlines areas where research evidence can support effective illegal wildlife trade policy, highlights critical uncertainties where research is required, and emphasizes the need for better design and evaluation of interventions that can help improve the effectiveness of efforts to combat illegal wildlife trade. Tools and expertise to improve the evidence base for national and international illegal wildlife trade policy already exist but are underutilized. Tapping into these resources could produce substantive benefits for wildlife conservation and associated sectors, enabling governments to fulfill their obligations under the Sustainable Development Goals and international biodiversity conventions. This could be achieved through enhanced funding support for intersectoral research collaborations, engaging researchers in priority setting and programme design, increasing developing country research capacity and engaging researchers and community voices in policy processes.

The Evidence to Action brief is the first of a new set of tools and guidance for researchers and practitioners. The latest addition is a brief reviewing the scale of Darknet Usage in the Illegal Wildlife Trade (osf.io/preprints/ socarxiv/fgrgd), which includes recommendations for researchers and policymakers. The darknet is a network of websites that can be accessed only via special software that hides the details of the user's connection, and allows websites to be hosted without revealing their location or operator. Large-scale darknet marketplaces exist for illegal drugs, firearms, hacking tools, stolen identity documents, and a wide variety of other illicit goods. However, the darknet has not, to date, proven to be an attractive platform for the buying and selling of illegal wildlife products (see also Oryx, 51, 393-394). Despite this, it provides a marketplace of last resort that becomes increasingly attractive over other, more accessible, online services as law enforcement and platform operators enforce policies against trading in illegal wildlife products. This makes the ongoing study of darknet markets an important avenue for research as other policies against online illegal wildlife trading emerge.

LAURE CUGNIERE (๑ orcid.org/0000-0002-4753-593X) Department of Zoology, University of Oxford, Oxford, UK E-mail laure.cugniere@zoo.ox.ac.uk

JOSS WRIGHT (๑ orcid.org/0000-0001-5237-3309) Oxford Internet Institute, University of Oxford, Oxford, UK

E.J. MiLNER-GULLAND (৫ orcid.org/0000-0003-0324-2710)
Department of Zoology, University of Oxford, Oxford, UK

\section{Halting the release of the pangolin Manis javanica in China}

As global travel and trade increase, so has the numbers of species spreading beyond their natural ranges. Introduction of alien species, intentionally or unintentionally, as a result of human activities may lead to the extinction of native species via interbreeding, predation, resource competition or the spread of disease. Pangolins are widely trafficked (S. Heinrich et al., 2017, The Global Trafficking of Pangolins: a Comprehensive Summary of Seizures and Trafficking Routes from 2010-2015, TRAFFIC South-east Asia, Selangor, Malaysia), and with the Chinese government improving wildlife protection and strengthening law enforcement the release of confiscated pangolins by law enforcement departments is increasing. There have been at least 10 cases of the release of confiscated pangolins into the wild in China since 2016, including one case each in October 2016, January, July and September 2017, May, June and August 2018, and three cases in July 2018 (mp.weixin.qq.com/s/zWQ27feivjiO4bBjZPKvFQ). In at least five cases the release was of Manis javanica, which is native to South-east Asia, including cases in Zhanjiang city, Hengyang city, Wenshan Zhuang and Miao Autonomous Prefecture, and Xishuangbanna Dai Autonomous Prefecture. 
Although releasing confiscated species into their natural habitats is potentially important for species conservation, releasing wild animals beyond their natural range is a complex undertaking that requires careful consideration and scientific support, without which release may not only fail to achieve the desired results but also harm the local ecosystem. In China M. javanica is native only in Menglian and Menghai counties, Yunnan Province (Wu et al., 2005, Acta Zootaxonomica Sinica, 30, 440-443). Most pangolins confiscated in China have been transported outside their range, from other regions or countries.

We believe that $M$. javanica should not be released into most regions of China, for the following reasons: (1) Most regions have a markedly different climate from that of the species' natural range, and pangolins have poor adaptability, impeding their survival outside their natural range $(\mathrm{Wu}$ et al., 2004, Chinese Journal of Applied and Environmental Biology, 10, 456-461). (2) M. javanica is a $K$-strategist, and thus the establishment of a sustainable population through the release of a few individuals outside their natural range is unlikely. (3) The species often carries multiple parasites or pathogens (Yang et al., 2010, Journal of Economic Animal [sic], 14, 22-25; Zhang et al., 2017, Zoo Biology, $36,387-396)$ and therefore release into new areas carries the potential risk of introducing parasites or pathogens. (4) As an alien species M. javanica may have adverse effects on local ecosystems through predation of, or competition with, native species such as Manis pentadactyla (Wu et al., 2005, Acta Zootaxonomica Sinica, 30, 440-443). (5) As the original source of confiscated pangolins is generally unknown, individuals may be released outside their source area, with the potential for genetic contamination of native populations.

Several Chinese institutions have made progress in conservation breeding research using confiscated $M$. javanica. The species survives well in captivity, and dozens of individuals have successfully mated and reproduced (Zhang et al., 2015, Folia Zoologica, 64, 129-135; 2017, Zoo Biology, 36, 387396). We recommend that the Chinese government establishes a pangolin rescue and breeding center for this rare species. Meanwhile, rescued individuals could be used to conduct conservation and biological research and to establish an ex situ population. In the future an ex situ population could be used for restoration of the wild population within its natural range.

FUHUA ZHANG (๑ orcid.org/0000-0002-5179-386X), YisHUANG $Y_{U}, W_{\text {ENHUA WANG, }} \mathrm{NA}_{\mathrm{X}} \mathrm{X}$ and SHIBAO WU (ㅇ orcid.org/0000-0003-4683-4919) School of Life Science, South China Normal University, Guangzhou, China. E-mailwushibao@163.com

\section{Discovery of a wild population of Orchidantha yunnanensis in south-east Yunnan, China}

The plant Orchidantha yunnanensis P. Zou, C.F. Xiao \& Škorničk of the family Lowiaceae was described based on living plants in Xishuangbanna Tropical Botanical Garden, Chinese Academy of Sciences, in 2017. The living plants were originally identified as Orchidantha chinensis T.L. Wu, collected from Malipo county in south-east Yunnan province in 2001. However, the authors recognized that the cultivated plants were not $O$. chinensis and they were therefore described as O. yunnanensis (P. Zou et al., 2017, Phytotaxa, $302,181-187)$. Orchidantha yunnanensis was thought to be extinct in the wild because no living plants were discovered during resurveys in the location of the original collection. The species may have been overcollected for medicinal uses.

During April 2018-March 2019 surveys were carried out in south-east Yunnan with the joint support of China's National Science and Technology Basic Resources Investigation Programme for Surveys and Germplasm Conservation of Plant Species with Extremely Small Populations in South-west China (grant no. 2017FY100100). Fifteen clumps of $O$. yunnanensis were discovered in one locality in Hekou Yao Autonomous County, c. $200 \mathrm{~km}$ from the original collection site. With such a small population size and with poor natural regeneration, O. yunnanensis needs to be categorized as a Plant Species with an Extremely Small Population (W. Sun et al., 2019, Trends in Plant Science, 24, 4-6). In April 2018 we collected vegetative material for propagation and ex situ cultivation in Kunming Botanical Garden; several plants have been successfully propagated and are growing well in a greenhouse.

Our survey, and information obtained from local people, indicated that the main threats to this species are habitat degradation and poor fruiting. The single known location of O. yunnanensis is not within a protected area. As the population is facing a high risk of extinction, protection of the known individuals and their habitat is critical, and research is also required on the reproductive biology and poor fruiting of the species. Yunnan Key Laboratory for Integrative Conservation of Plant Species with Extremely Small Populations and South China Botanical Garden, Chinese Academy of Sciences, are now cooperating to study these matters. Further surveys for the species are also required in the karst region of south-east Yunnan and the adjacent area of northern Viet Nam.

\footnotetext{
Lei CaI, Zhiling Dao and WeIbang Sun Yunnan Key Laboratory for Integrative Conservation of Plant Species with Extremely Small Populations, Kunming Institute of Botany, Chinese Academy of Sciences, Kunming, Yunnan, China E-mailwbsun@mail.kib.ac.cn
} 